\title{
Developing Al-Najaf Cement Plant using Wireless SCADA System
}

\author{
Mehdi J. Marie \\ Dept. of Electrical Eng. \\ College of Engineering \\ University of Basrah, Iraq
}

\author{
Ghaida A. AL-Suhail \\ Department of Computer Eng. \\ College of Engineering \\ University of Basrah, Iraq
}

\author{
Hussein T. Kadhim \\ Dept. Automation \& Systems \\ Research \& Development Dir. \\ Ministry of Science and Tech.
}

\begin{abstract}
Wireless technologies are rapidly being adapted from simple monitoring and control to supervisory control and data acquisition (SCADA) system. Wireless provides highly reliable data communications in harsh and interference-heavy environment. In this article, new wireless SCADA system has been built to control Al-Najaf cement plant wirelessly. Two pieces of nanostation5 $(5 \mathrm{GHz})$ which utilize the Wi-Fi wireless technology and IEEE802.11/g standard were used. They used as transceiver for transmitting and receiving the information between master station (MS) and remote terminal units (RTUs). CIMON devices from KDT systems for experimental set-up were used. A more detailed focus on Iraq cement plants using wireless technologies for monitoring and control of the whole process as an application also was presented.
\end{abstract}

\section{General Term}

Computer networks and protocols, Computer control and systems, Industrialized embedded control systems, computerized control and automation, Wireless sensor networks.

\section{Keywords}

Wireless SCADA systems,Zigbee, WiFi, PLC, KDT systems, RTU, MS, cement plant.nanostation5,

\section{INTRODUCTION}

The motivation for this work is to support the work in Iraqi cement plants refurbishment. They had been installed more than thirty years ago. The monitoring and control equipments all over the factories are old and if new equipment is purchased, it is not possible to use full benefit of its capabilities. They are run with less than their production capacity. There are a lot of pollutants released from cement plant such as $\mathrm{Co}_{\mathrm{x}}, \mathrm{So}_{\mathrm{x}}, \mathrm{No}_{\mathrm{x}}$ and high quantities of dust. A reliable wireless monitoring system is also required to limit the harmful of these hazardous gases. Al-Najaf cement plant is the oldest and smallest between factories to be studied. It has only one rotary kiln and hence only one production line. All cement plants are installed on a large area. The units are distributed and there is a large distance between them. Management task for all over the plant requires a new modern reliable system with low cost. When compared with traditional cable based circuits, wireless technology offers advantages, including increased flexibility, easy installation, and cost savings.

SCADA system nowadays enters in every establishment even it is simple facility such as small sewage treatment plant to large facilities such as national grid. It offers a lot of features such as sensing, monitoring, communication, control, maintenance reports and scheduling times. For the best of author knowledge, this paper is the first that addresses the use of nanostatio5 as a transceiver for control in the SCADA systems. A successful implementation of an experiment for controlling the level and temperature wirelessly using nanostation 5 for data exchange has done.

The rest of this paper is organized as below. Section two is devoted to related prior works in the field of SCADA systems. Fundamentals of SCADA system with related fields are explained in section three and its associated subsections. Section four discussed the application of SCADA system for Al-Najaf cement plant and its different RTUs. The experimental set-up and the devices that have been used are investigated in section five, while section six summarized the main conclusions of this article.

\section{RELATED WORK}

Schlechtingenet al [1] proposed a system for wind turbine condition monitoring using adaptive neuron-fuzzy interference systems (ANFIS). For this purpose, ANFIS normal behavior models for common SCADA data are developed in order to detect abnormal behavior of the captured signals and indicate component malfunctions or faults using the prediction error. A new conceptual design of an intelligent SCADA with a decentralized, flexible, and intelligent approach, adaptive to the context (context awareness) was proposed by Vale et al [2]. This SCADA model is used to support the energy resource management undertaken by a distribution network operator.

In [3], Otaniet al proposed a SCADA system using mobile agents for flexibility. In addition, they show two types of communication protocols that make agent migration more fault-tolerant, and perform experiments where the SCADA system executes earth fault protection within the required time.De Oliveira et al [4] presented a new load flow formulation to solve active and passive electric distribution networks. The fundamental idea discussed there is how to obtain the power flow solution by using the elements of a unique quasi-symmetric matrix in the iterative process. Figueiedoet al in [5] developed an energy management platform for intelligent buildings using a SCADA system. This SCADA system integrates different types of information coming from the several technologies present in modern buildings (control of ventilation, temperature, illumination, etc.) while Loukah and Jibra [6] recalled the basic concepts of SCADA systems, to present the project management phases of SCADA for real time implementation, and then to show the need of the automation for electricity distribution companies on their distribution networks and the importance of using computer based system towards sustainable development of 
their services. A proposed computer based power distribution automation system is then discussed. Finally, some projects of SCADA system implementation in electrical companies over the world are briefly presented.

Roshanet al [7] mainly focused on proper coordination of heater and supporting components in order to reduce its energy consumption and optimize power for specific input parameters. They implemented PLC based controller to handle any type of high and medium capacity heater system. Lakshmi et al [8] presented the experimental validation procedure of a simple cascade control system through number of architectures, such as SCADA, PLC and internet. The performance and effectiveness of individual architecture is evaluated on the basis of data rate, rise time, peak time and settling time.

Caiet al [9] suggested a reliable remote control system for subsea blowout preventer stack which has been developed based on the off-the-shelf triple modular redundancy system. To meet a high reliability requirement, various redundancy techniques such as controller redundancy, bus redundancy and network redundancy are used to design the system hardware architecture. Biswalet al [10] presented a development and implementation of SCADA based process control and monitoring system. It proposes a novel six stage standbys redundant structured for generators cooling in a typical thermal power plant.

Ahiskaet al in [11] have been carried out new test measurement system for supervisory control and data acquisition application with PLC. During the production of the electric energy from the thermoelectric generator, the temperatures of the surfaces of the thermoelectric generator, current-voltage values obtained from output of thethermoelectric generator, hot and cold flows have been measured by the newly established system. Cetincevizetalet al [12] described an Internet based control system with wireless fieldbus communication designed for distributed processes. The system was implemented as an experimental setup in a laboratory.

\section{FUNDEMENTALS OF SCADA SYSTEM}

SCADA system is that one which interested in collecting data from field using different devices such as sensors, computers, servers, switches, PLCs etc. It is standard since 1962 [13] where before that time there was a difficult for doing this task. The plant is divided to different group of RTUs for each production stage. The RTUs are monitored and controlled by MS which contains the main display units and software. The connection between MS and RTUs is done through a communication link. It may be a wired line, wireless or a hybrid wire/wireless links. The SCADA system depends on three fields of electrical engineering, communication, computation, control (CCC) or $\left(\mathrm{C}^{3}\right)$.

\section{SCADA MASTER}

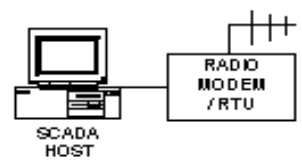

\section{REMOTE SITE}

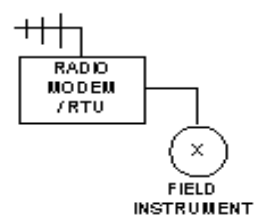

\subsection{Wireless Communication}

Wireless technologies are increasingly being developed to be used in simple applications to large SCADA systems. Wireless networks can provide highly reliable data communication in hazard and interference-heavy environments. Figure 1 illustrates a schematic diagram of wireless SCADA system. Compared with traditional cable based circuits, wireless technology offers advantages, including increased flexibility, easy installation and cost savings. All wireless systems operate on some frequency, communicate some distance and offer some data rate. These factors affect each other, so it is important to find a compromised method to satisfy the requirements for a certain application [14].

The identifier for radio is the frequency. There are three frequencies, $900 \mathrm{MHz}, 2.4 \mathrm{MHz}$ and $5 \mathrm{GHz}$ represent the Industrial, Science and Medicine (ISM) bands. In radio network, once a signal has left the antenna and communicated over the air, energy continues to be lost over the communication path which is a function defining lost energy at frequency over a distance. As the frequency and distance increase, then free space loss does [10].

Another aspect of communication is the data rate. A radio does not operate at one frequency; instead it does in a band of frequencies. This band can be used in its entirety or in smaller sections, typically called channels. The spectrum that the radio operates in (weather it is an entire band or specific channels) is considered radio's channel bandwidth. A high speed radio uses a wider channel bandwidth regardless of the transmission method. Channel bandwidth refers to the amount of data that can be transmitted by radio signal. It is measured in bytes transferred over a period of time (Kbps or Mbps). Higher speed communication requires a wider bandwidth making high speed radios more susceptible to interference.

There are different data transmission modes [15]. They have various unique characteristic. These variations result in the ability to communicate different amount of data at varying distances. Table A-1 in Appendix A shows wireless technologies used by SCADA system. One or more of the following transmission methods can be used in each of the wireless technologies:

\section{- Fixed frequency}

- Frequency hopping spread spectrum (FHSS)

- Orthogonal frequency- division multiplexing

(OFDM)

- Direct sequence spread spectrum (DSSS)

\subsection{Computation and Software}

In SCADA system computation and networking jobs are done by computers abilities such as, processing and saving of high quantity of data. In MS there is a main computer that monitors and controls the whole process with a back-up one. Operation manager can make setting of different devices. A decision can be issued to change the flow of the process as required according to some desired states and control algorithms. A lot of software packages have been developed like the one we used to build ladder diagrams CICON and CIMOND for building SCADA systems.

Fig 1: Wireless connection between MS and RTU 


\subsection{Control Algorithms}

The control algorithms and methods can be applied to the SCADA system [5]. The MS and RTUs can implement the PID, LQR, Fuzzy logic control [1] to lead the process flow for some desired states and required parameters. Methods for optimization such as genetic algorithm and particle swarm optimization also can be used. These strategies can be implemented in devices such as smart relays, PLCs, PCs that found in different SCADA subsystems.

\subsection{SCADA Subsystems}

The main SCADA subsystems can be listed as follows

- A communication network connecting the MS with RTUs. It may be wire or wireless network.

- MS which is the supervisory system responsible for receiving packets of information from RTUs and send decisions and reports for maintenance, scheduling and alarms.

- RTUs consist of one or more PLCs which connect directly to the field of the process. Each separate unit can be regarded as RTU.

- A mimic or human machine interface (HMI) which displays the information to the operation manager.

\subsection{Wireless SCADA Architecture}

The typical wireless SCADA architecture (see appendix B) is Point-Multipoint with one Master polling multiple RTUs. Using RTU or PLC wireless data communication protocols including protocols such as Modbus, Wi-Fi, wireless Ethernet, Zigbee, etc. [17]. Each PLC or RTU at the remote site is programmed with a unique system address and those addresses are all configured into the SCADA Host HMI. The SCADA Host then polls these addresses and stores the acquired data into its database. It will perform centralized alarm management, data trending, operator display and control. Modern SCADA Host HMI's like Net SCADA can easily accommodate many different types of industrial protocols and the architecture allows multiple clients to view the same data and seamless expansion to handle additional remote sites and I/O points. Bentek systems [18] provides complete turnkey SCADA and Wireless SCADA communications solutions from the SCADA Host HMI to the Satellite or Cellular communications or Private Radio communications. Wireless SCADA systems can range from simple Point-Multipoint systems like the 900-MB Spread Spectrum system below using Modbus protocol as shown in Figure 2 (a). Figure 2 (b) depicts the SCADA systems for AlNajaf cement plant.

\section{SCADA SYSTEM BASED - CEMENT PLANT}

In Al-Najaf cement plant, SCADA system is designed as shown in figure 2 (b) [13], there are 12 RTUs and one MS. The communication between them is done by wireless link. The following stages are basically found in most cement plants [19]:

RTU\#1. Quarry: Limestone is blasted from the quarry site. The high investment in new technology insures that the rock fragmentation is constant in size. It is then loaded onto dumpers and taken to modern crushing and screening machinery.

RTU\#2. Crushing: Any combination of five separate crushing installations is used, depending on the raw material type and source, to provide properly sized materials for feeding into the raw mill.

RTU\#3. Blending:Carefully proportioned quantities of each material are fed by conveyor belt to the Pre blending.

RTU\#4. Quality Control:The quality of the materials at all stages in the process is closely monitored. From the raw materials in the quarry through to the finished cement, chemical and physical characteristics are assessed and adjusted by self-regulating automated systems. This high degree of quality control ensures reliability in the final product.

RTU\#5. Additive Silos:Four large additive bins are used for addition of limestone, and five further additive bins are available when required for adding small volumes of materials to fine-tune the chemical parameters of the raw-mix.

RTU\#6. Raw Mill: The Atox type vertical raw mill, crushes and grinds raw materials to a very fine powder, known as raw meal. The raw meal is then separated out of the air stream by a system of cyclones, before it is brought to the Silos.

MS:The entire operation is carefully monitored in the MS. Several thousand parameters affecting the plant are monitored, fed back and displayed on screens in the Control Room. From here operators can monitor the performance of the process control systems and intervene where necessary to ensure process stability and product quality.

RTU\#7. Rotary Kiln:The kiln is really the heart of the whole process, because here the material temperature is raised from 860 to 1500 deg $\mathrm{C}$, thereby enabling the chemical reactions which form clinker to take place.

RTU\#8. Cooler:The purpose of the Grate Cooler is to cool the clinker after it exits the kiln, recuperating as much heat as possible and return it to the calciner, thereby reducing overall heating costs. Oversized clinker is crushed before being transported along with the other clinker, to the clinker storage shed by bucket conveyor.

RTU\#9. Storage:This concrete storage shed has the capacity to hold thousands of tonnes of clinker. Any combination of nine extraction points can be used to extract clinker to feed the cement mill.

RTU\#9. Electrostatic Precipitator: The precipitator is used to purify exhaust gases. The dust particles become electrically charged and attach themselves to plates inside the precipitator. The plates are cyclically rapped and the discharged dust is collected.

RTU\#10. Fuel Tanks: It consists of tanks (contains the crude oil), pumps, heaters, different sensors and controllers.

RTU\#11. Electrical Substation: It consists of transformers, high voltage breakers, monitors and controllers.

RTU\#12. Fire Fighting System: Smoke sensors, water pumps and controllers are the main components here. 


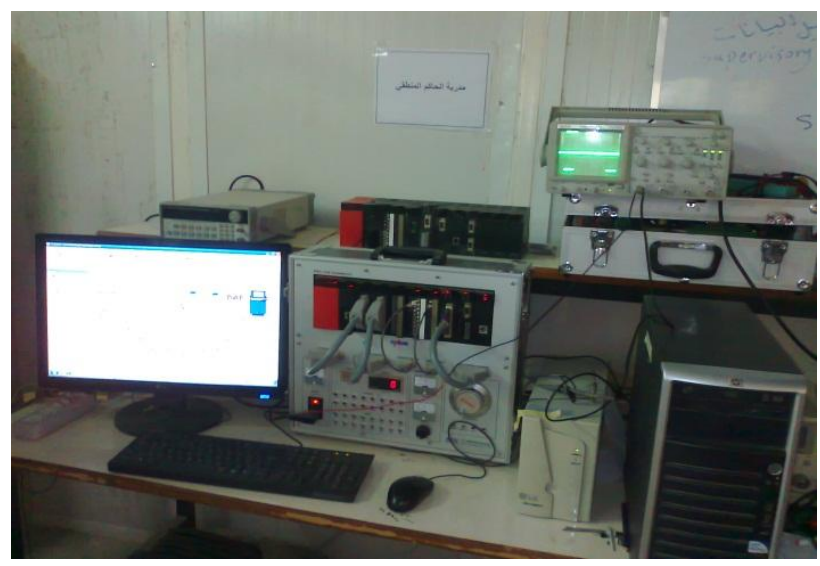

(a)

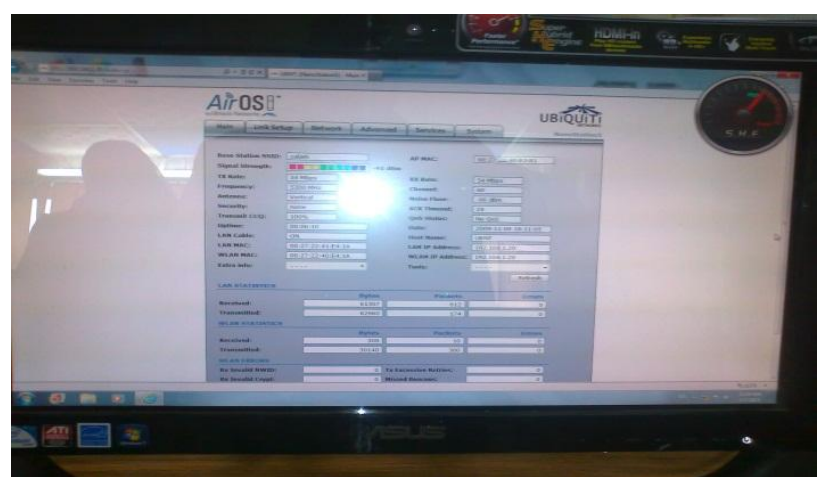

(c)

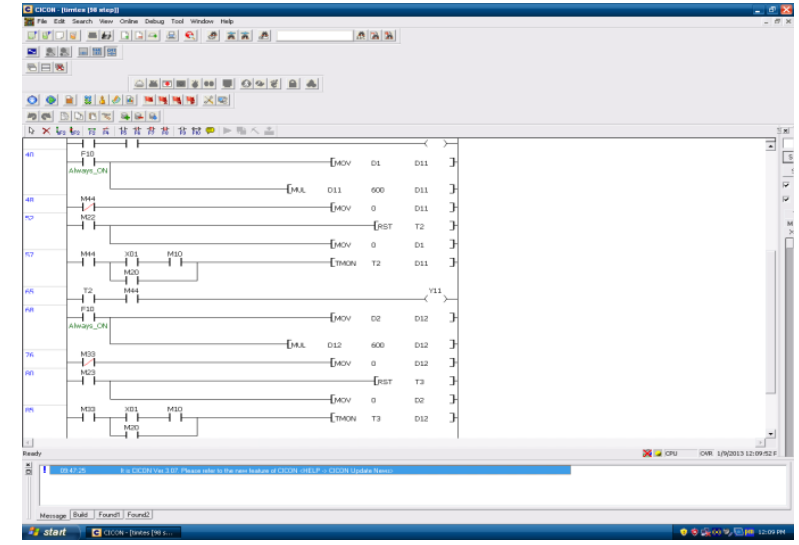

(b)

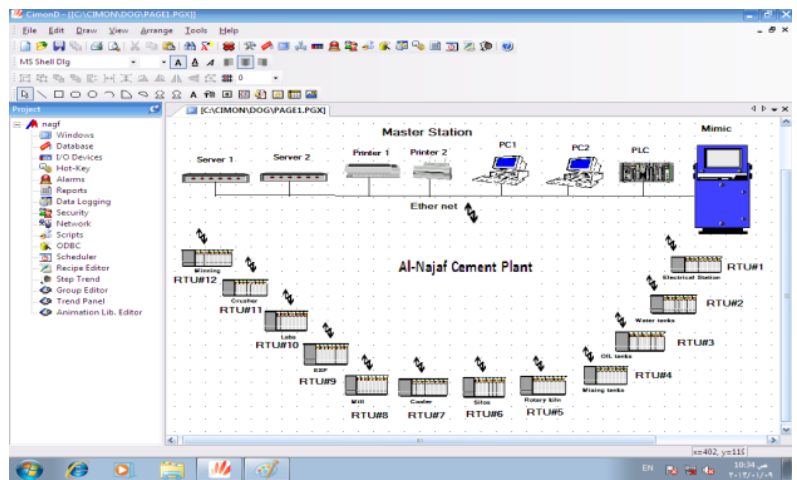

(d)

Fig 2: Experimental set-up of SCADA system (a) Devices of the experiment in the SCADA lab.; (b) Ladder diagram implemented in CIMON PLC; (c) Setting of the nanostation5 parameters; (d) Designing of SCADA system for Al-Najaf cement plant using CIMOND software package, one MS and 12 RTUs.

\section{EXPERIMENTAL SET-UP}

We designed the wireless SCADA system for Al-Najaf cement plant in the SCADA laboratory of the department of Automation and Systems at Ministry of Science and Technology with the following steps

- Divide the main units of the plant to 12 RTUs and one MS as can be shown in fig.2 (d).

- Each RTU contains a PLC where input/output points are determined for sensors and actuators.

-The MS unit contains two PCs, servers, printers and PLC; one active and the other is back-up.

-The wireless connection between each RTU and MS is implemented by using the nanostation $5(5 \mathrm{GHz})$. It used the WiFi technology with IEEE802.11 standard [20]. The software that has been used for building the Ladder programs is CICON from KDT systems [21], While the HMI diagrams are built using CIMOND program from the same company.
The results obtained from the practical experiments are the responses of the control system for a reference input as shown in figure 3 (a) and (b) respectively. The simulation results are obtained using the Truetime 2 beta6 simulator [22].Figure 3 (c) represents the wireless control system for rotary kiln using WiFi network, while (d) is the step response for the closed system. The transfer functions for the actuator (which is composed of burner and motorized valve), rotary kiln and thermocouple sensor are derived following the same procedure in [23].The transfer function of the closed loop control system is

$\frac{T_{o}}{T_{r}}=\frac{0.3 S^{2}+0.6 S+0.1}{S^{3}+0.4 S^{2}+0.6 S+0.1}$

and of PID controller as

$\frac{\mathrm{U}(\mathrm{S})}{\mathrm{E}(\mathrm{S})}=\mathrm{K}_{\mathrm{p}}\left(1+\frac{1}{\mathrm{~T}_{\mathrm{i}} \mathrm{S}}+\mathrm{T}_{\mathrm{d}} \mathrm{S}\right)(2)$ 


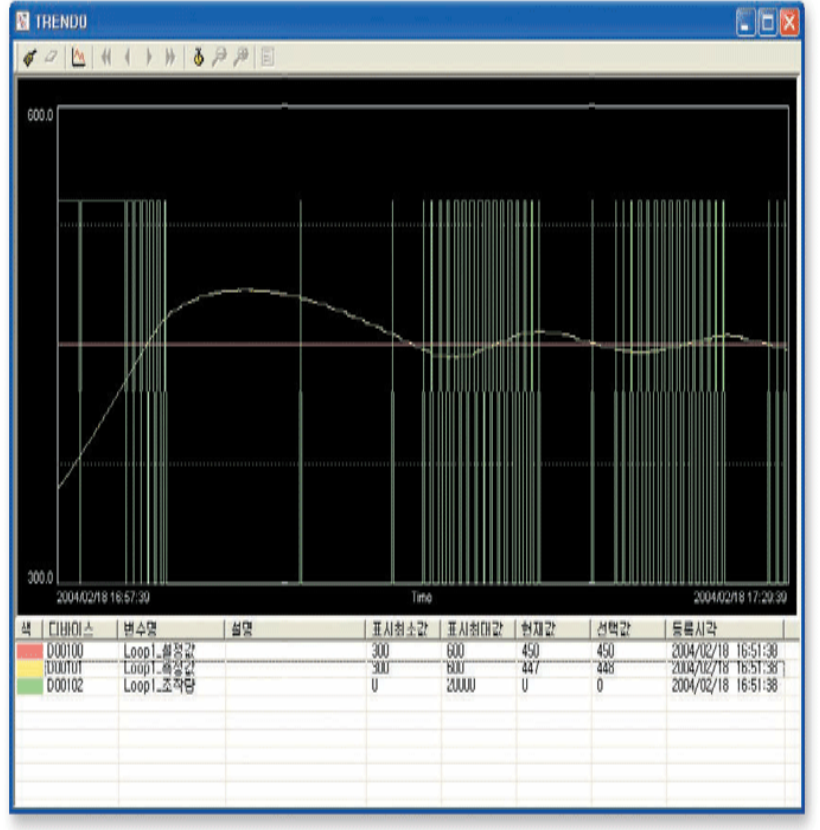

(a)

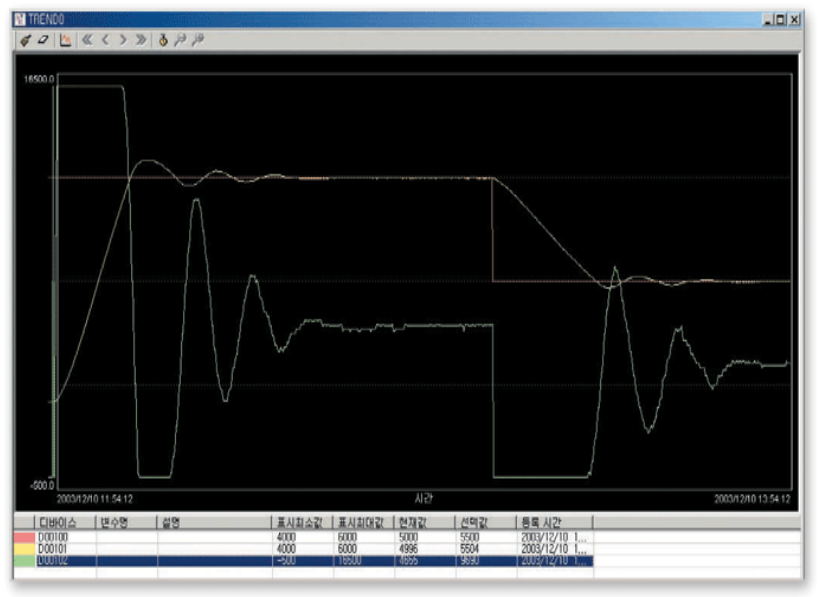

(b)

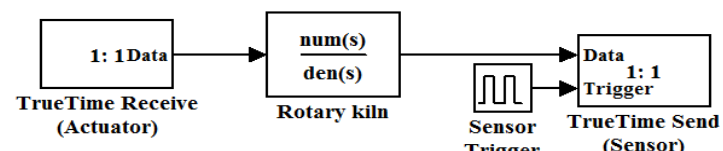
$\begin{array}{ll}\begin{array}{l}\text { Sensor } \\ \text { Trigger }\end{array} & \text { (Sensor) }\end{array}$
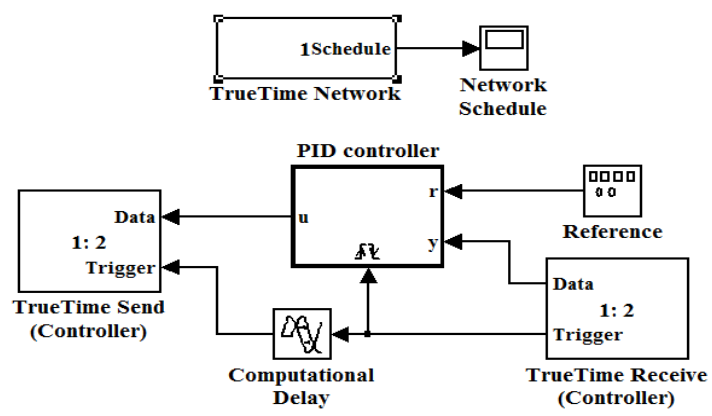

(c)

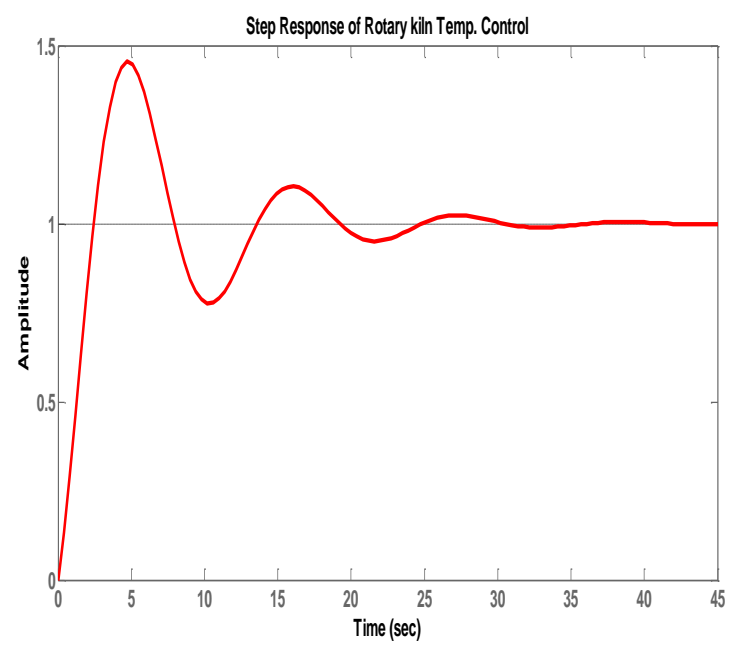

(d)

Fig 3. Implementation of PID Controller through Wireless SCADA system; (a) Response with Scheduling of On-Off Operation; (b) Response of the Wireless Control System without Scheduling; (c) Rotary kiln wireless control system using Truetime 2 beta6 simulator; (d) Step response of the system.

where $T_{0}$ : output temperature of the kiln

$\mathrm{T}_{\mathrm{r}}$ : required temperature for the kiln

$\mathrm{u}$ : control signal

e : error signal

$\mathrm{K}_{\mathrm{p}}$ : proportional gain

$\mathrm{T}_{\mathrm{i}}$ : the integral time

$\mathrm{T}_{\mathrm{d}}$ : derivative time

The tuning of PID controller parameters is done by using the well-known Zeigler-Nichols method as below

$\mathrm{Kp}=26.144 ; \mathrm{Ti}=3$ second $; \mathrm{Td}=0.75$ seconds;

\section{COMPARISONS WITH PRIOR WORK}

The results that have been obtained from using nanostation5 in the control applications are compared with that of [10] and [11]. In [10], the results for controlling the hydrogen cooling control system is compared with that of controlling the rotary kiln in cement plant. In spite of that a wireless control system is used in this paper, the results seems to be promising. The response of the closed loop with PID controller is acceptable, since it has low rise time, fair overshoot, low settling time, etc.

The results obtained in this paper are also compared with that of [11]. A control system is designed to control the thermoelectric generator. They used a programmable logic controller to implement the PID controller. In this paper, the PID controller is implemented using the simulation methods. The results are approximately identical.

\section{CONCLUSIONS}

In this paper, we have tried to contribute the efforts to refurbish the Iraqi cement factories. The wireless control system is suitable for our environment because there is always 
a dusty weather and a lot of pollutants were there, so the maintenance is very difficult. The wireless SCADA system if it is decided to be used will be very useful to overcome problems and it can be regarded as the best solution. The use of nanostation5 for data transmission between the MS and RTUs is implemented practically for controlling the temperature of rotary kiln and level in tanks. The practical and theoretical results are compared. The quality and quantity of the product will be enhanced accordingly using wireless SCADA system.

\section{ACKNOWLEDGMENTS}

Our thanks and appreciations to the engineers and technicians of the wireless SCADA laboratory at the Iraqi Ministry of Science and Technology who have contributed towards development of this work.

\section{REFERENCES}

[1] M. Schlechtingen, I. Santos and S. Achiche, "Wind turbine condition monitoring based on SCADA data using normal behaviour models. Part1: System description," Elsevier, Journal of Applied Soft Computing, Vol.13, Issue1, 2013, pp. 259-270.

[2] Z. Vale, H. Morais and C. Ramos, "Distribution system operation supported by contextual energy resource management based on intelligent SCADA," Elsevier, Journal of Renewable Energy, Vol.52, 2013, pp.143-153.

[3] T. Otani and H. Kobayashi, "A SCADA system using mobile agents for a next generation distribution system," IEEE Transaction on power Delivery, Vol.28, Issue 1, 2013, pp.47-53.

[4] P.M. De Olivera, M.A. Alvarez and J.M. Yusta, "Distribution flow method based on a real quasisymmetric matrix," Elsevier, Journal of Electric Power system, Vol.95, 2013, pp148-159.

[5] J. Figueredo and J. Sada Costa, "A SCADA system for energy management in intelligent building," Elsevier, Journal of Energy and Buildings, Vol.49, 2012, pp85-98.

[6] M. Loukha and M. Jibra, "Project management phases of a SCADA system for automation of electrical distribution network," International Journal of Computer Science Issue, Vol.9, Iss.2, 2012, pp157-162.

[7] B. Roshan, and K. Pravin, "Optimization of energy for industrial heater using PLC and SCADA," International Journal of Computer Technology and Electronic Engineering, Vol.2, Issue 1, 2012, pp200-204.

[8] A. Lakshmi, B. Naveenkumar, A. Balji and N. Bharathi, "Experimental validation of PID based cascade control system through SCADA-PLC-OPC and internet architecture," Elsevier, Journal of measurements, Vol.45, Iss.4, 2012, pp643-649.

[9] B. Cai, Y. Lui, Z. Lui, Z. and F. Wang, "Development of an automatic subsea blowout preventer stack control system using PLC based SCADA," Elsevier, Journal of ISA Transaction, Vol.51, Issue 1, 2012, pp198-207.

[10] G.R. Biswal, R.P. Maheshwari, and M.L. Dewal, "Dynamic process control and monitoring of novelS3RS based hydrogen cooling system," Elsevier, Journal of Electrical power and Energy Systems, Vol.43, Issue 1, 2012, pp. 162-172.

[11] R. Ahiska, and H. Mamur, "A test system and supervisory control and data acquisition application with programmable logic controller for thermo electric generators," Elsevier, Journal of Energy Conversion and Managements, Vol.64, Iss.1, 2012, pp15-22.

[12] Y. Cetinceviz, and R. Bayindir, “ Design and implementation of an internet based effective controlling and monitoring system with wireless field bus communications technologies for process automation- an experimental study," Elsevier, Journal of ISA Transaction , Vol.51 Iss.3,2012,pp461-470.

[13] SCADAHistory,Http://wargtachinic.blogspot.com/200g/ 03/scada.html.

[14] D. Bailey," Practical SCADA for industry", Newens, 2003.

[15] DPS Telecommunications, "Wireless Ethernet," White paper, www.dpstele.com.

[16] "White papers"www.emersonprocess.com/smart wireless

[17] BENETEK systems, www. scadalink.com

[18] Technical Reports ,'Programming of SCADA system and PLC," Http://www.citect.com

[19] Quinn cement group," Technical manual,” www.quinngroup.com.

[20] Technical Manual, www.ubnet.com

[21] Technical Reports, www. KDTsystems.com

[22] D. Henriksson, M. Ohlin, and A. Cervin," Truetime simulations of networked computer control systems," in $2^{\text {nd }}$ IEAC conference on analysis and design of hybrid systems, 2006.

[23] R. Burns," Advanced control engineering," ButterworthHeinemann press, Oxford, 2001. 


\section{Appendix A}

Table 1. Short range wireless SCADA [16]

\begin{tabular}{|c|c|c|c|c|c|}
\hline Property & \multicolumn{2}{|c|}{ WUSB ${ }^{1}$ BLUETOOTH } & \multicolumn{2}{|c|}{ Wi-Fi ${ }^{2}$ WiMAX } & Zigbee \\
\hline Range (m) & 10 & 10 & $50 \mathrm{a}-100 \mathrm{~b} / \mathrm{g}$ & up to 2000 & $10-100$ \\
\hline Frequency $(\mathrm{GHz})$ & 2.4 & 2.4 & $2.4-5.4$ & 5.8 & 2.4 \\
\hline Rate (Mbps) & 0.62 & 1 & $11-54$ & 75 & 20 \\
\hline Power & Low & Medium & High & High & $0.2-0.4-2.5$ \\
\hline Complexity & Low & Low & High & High & Very Low \\
\hline Application & $\mathrm{PC}^{3}$ & $\mathrm{PDA}^{4}$ & PC, Security & --- & Low \\
\hline SCADA suitability & Low I & & Medium Me & & High \\
\hline
\end{tabular}

1. Wireless universal serial board

2: Wireless - Fidelity

${ }^{3}$ : Personal computer

${ }^{4}$ : Personal digital assistant

\section{Appendix B}

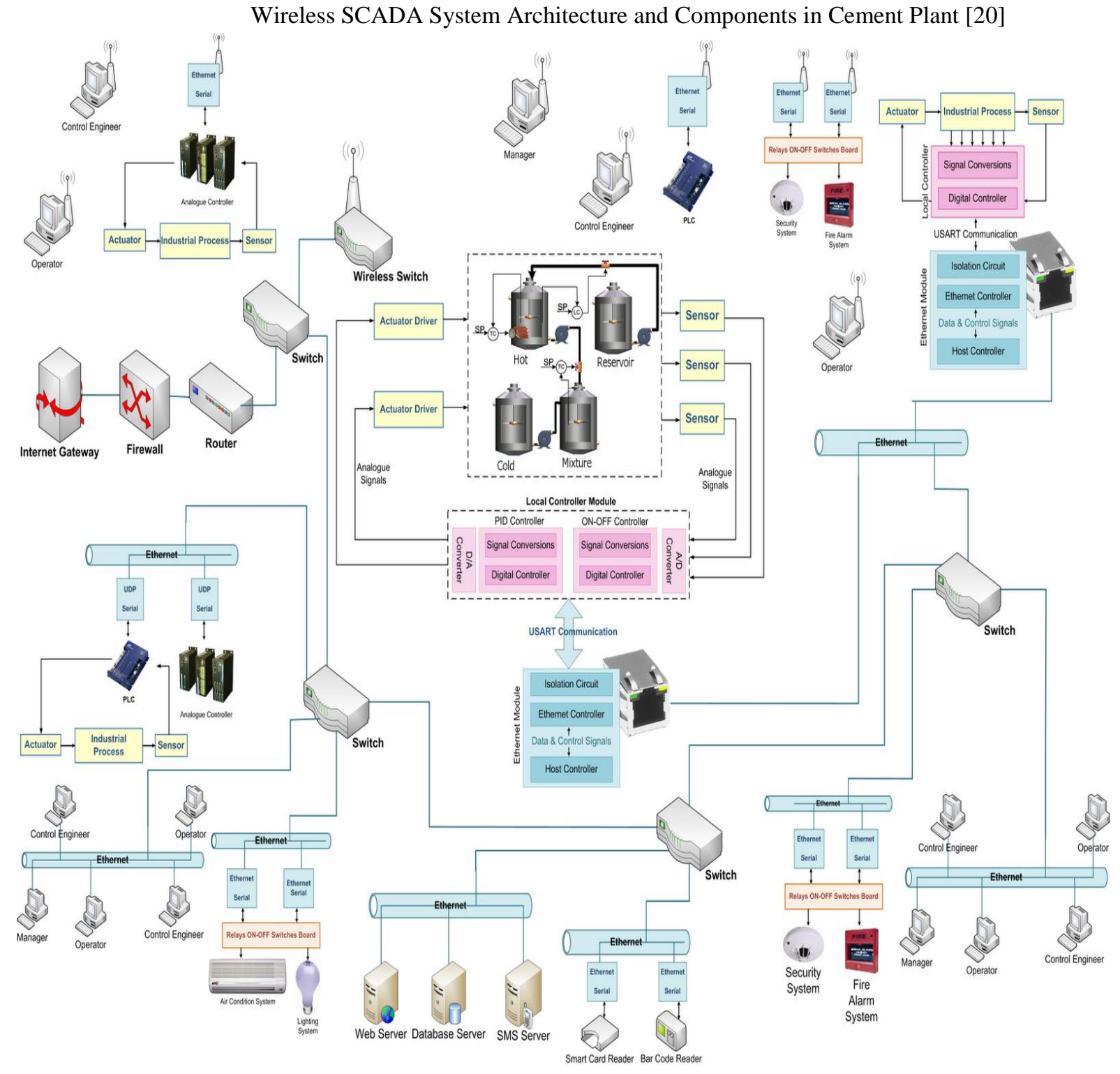

